\title{
A Review of Progress in Modelling Induced Geoelectric and Geomagnetic Fields with special regard to Geomagnetically Induced Currents
}

\author{
Alan W. P. Thomson ${ }^{1}$, Allan J. McKay ${ }^{1}$ and Ari Viljanen ${ }^{2}$ \\ 1 British Geological Survey, Murchsion House, West Mains Road, Edinburgh, EH9 3LA \\ awpt@bgs.ac.uk, aljmebgs.ac.uk \\ ${ }^{2}$ Finnish Meteorological Institute, Helsinki, Finland. ari.viljanen@fmi.fi
}

Summary. The Earth's lithosphere and mantle responds to Space Weather through time-varying, depthdependent induced magnetic and electric fields. Understanding the properties of these electromagnetic fields is a key consideration in modelling the hazard to technological systems from Space Weather. In this paper we review current understanding of these fields, in terms of regional and global scale geology and geophysics. We highlight progress towards integrated European-scale models of geomagnetic and geoelectric fields, specifically for the purposes of modelling geomagnetically induced currents in power grids and pipelines.

\section{Introduction}

Geomagnetically Induced Currents (GIC) flow in grounded conducting networks, such as power grids and pipelines, during geomagnetic storms. GIC are near-DC electrical currents that are a consequence of the induced geoelectric field that follows from Faraday's law of electromagnetic (EM) induction. To fully understand the flow of GIC in networks we need to first understand how the geoelectric field responds to a given geomagnetic disturbance. This geophysical response depends on three main factors: the spatial structure and variation periods of the primary geomagnetic field and the three-dimensional conductivity structure of the Earth. Given a surface distribution of the geoelectric field, electrical network analysis can then be used to determine the flow of GIC in conducting networks (e.g. Lehtinen and Pirjola, 1985; Pulkkinen et al., 2001).

In this review we will report on recent progress on the geophysical problem, particularly in the understanding of the three-dimensional electrical conductivity structure of the Earth from both global and regional EM surveys, and from technical (modelling) innovations. We summarise major recent discoveries and provide, for the reader, references to the major papers. We concentrate on papers published in the last ten years or so and therefore refer the reader to the reviews of Schwarz, 1990, and Hjelt, 1988, for the status of the scientific literature prior to this time.

The structure of the paper is as follows. In Section 2 we discuss recent global induction studies that reveal current 'best estimates' of the electrical conductivity of the Earth's mantle 
and lithosphere. In Section 3 we highlight studies that provide resources relevant to deriving regional conductivity models, for example on the European continental scale. In Section 4 we outline various techniques that are, or could be, employed to model the EM fields relevant to GIC. Note that the scope of this paper is restricted to the electromagnetic induction studies relevant to GIC, and is not intended to be exhaustive. The reader is referred to Kuvshinov (2007) for a review of induction effects (in 3-D and 1-D models at sea level and satellite altitude) in magnetic fields due to magnetic storms, geomagnetic daily variations, tides and ocean circulation.

\section{Global Conductivity Models}

The Earth surface 'footprint' of Space Weather can be large: both continental-scale and globalscale EM fields can be induced in the Earth, depending on the scale size and period of external magnetic variations, these being subject to solar wind control. The global scale response occurs primarily in the deep mantle. In this section we highlight recent global induction studies that provide deep mantle conductivity models. These models typically have a simple radial dependence and are used to underly various crustal/ upper mantle models required to model EM fields at periods relevant to GIC.
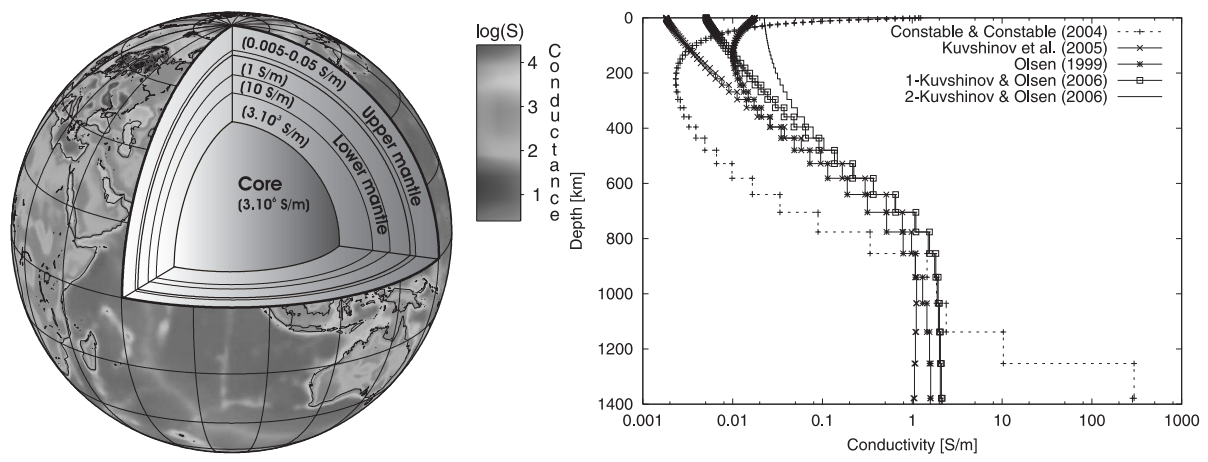

Fig. 1. Left - Global map of surface conductance with the cutaway showing the Eurasian mantle conductivity model of Semenov and Jozwiak (1999); after Vozár et al. (2006). Right - Mantle conductivity profiles of various studies; see Kuvshinov and Olsen (2006) for full details and references.

\subsection{Radial Conductivity Models}

Magnetic satellite missions such as CHAMP (2001 to present), Ørsted (1999 to present), and SAC-C (2002 to present) offer global uniform data coverage. These data sources have been used to derive deep (to a depth of $1500 \mathrm{~km}$ ) radial conductivity models (e.g. Kuvshinov and Olsen, 2006). At depths greater than $400 \mathrm{~km}$ the models consistently show a monotonic increase in conductivity from about $0.03-0.08 \mathrm{~S} / \mathrm{m}$ to $1-2 \mathrm{~S} / \mathrm{m}$ at $900 \mathrm{~km}$ depth; see Figure 1 for example. 


\subsection{Three Dimensional Conductivity Models}

An inhomogeneous distribution of electric conductivity in the Earth's crust will distort even long period EM field variations that penetrate deep into the Earth. Therefore, to model properly the geoelectric and geomagnetic field 3D inductive effects must be taken into account. For example, $3 \mathrm{D}$ analysis is required to interpret satellite induction observations in the longer period range of hours to days (e.g. Everett et al., 2003). Similarly, long period electromagnetic responses, say derived from geomagnetic observatory data, need to be corrected for the distorting effect of induction in the oceans.

For example, Olsen and Kuvshinov (2004) used a global surface conductance (conductivitythickness product) map (S-Map) (see Figure 1) to model the ocean effect of geomagnetic storms. The S-Map represents the non-uniform surface conductance due to the seas, oceans and sediments and are often formulated using a combination of bathymetric data, and the global sediment thickness compilation of Laske and Masters (1997); see Everett et al. (2003) for example. However, Vozár et al. (2006), for example, are refining a global S-map using a combination of Magnetotelluric (MT) and Geomagnetic Deep Sounding (GDS) data. If successful, this will prove to be a valuable resource as it will draw together information gained from both global and regional induction studies.

Compilations such as the world geological map (http://ccgm.free.fr) and world magnetic anomaly map (Purucker, 2007) provide insights into global (and regional) tectonics. The planned ESA Swarm mission should provide information about electrical conductivity heterogeneities in the Earth's mantle (Kuvshinov et al., 2006)

\section{Regional Conductivity Models}

The main sources of regional conductivity models stem from MT and GDS surveys; a comprehensive review of which is outwith the scope of this paper (but see Haak (1985) for an earlier review, continent by continent). We restrict ourselves therefore to a summary of regional conductivity studies of relevance to modelling GIC within Europe and North America. However we note that studies reported in Schwarz (1990) provide valuable detail for regions such as New Zealand, South Africa, Australia, and Japan.

Recent European investigations of the electrical conductivity of the lithosphere and asthenosphere have been reviewed comprehensively by Korja (2007). Fennoscandia is wellcovered in respect of recent MT and GDS studies following the completion of the Baltic Electromagnetic Array (BEAR) project. Korja et al. (2002) compiled a map of the crustal conductance for the Fennoscandian Shield and its surrounding ocean and seas, and continental areas using the BEAR array data, and numerous other studies. The crustal conductance compilation of Korja et al. (2002) has been utilized by Engels et al. (2002) to model electric and magnetic fields at the Earth's surface.

The electrical conductivity structure of the UK landmass is complex and it is surrounded by shallow shelf seas along with the deep ocean a few hundred kilometers to the west. These factors are all known to influence the EM fields observed on land in a period range appropriate to GIC (McKay and Whaler, 2006). Thus, the geological setting, and geomagnetic latitude, of the UK has influenced the approach to modelling the EM fields. For example, Thomson 
et al. (2005) used a quasi 3D thin-sheet model to calculate the geo-electric field at the peak (as determined by the time of maximum GIC in the Scottish Power grid) of the October 2003 geomagnetic storm; see Figure 2. They noted the regional variation of the electric field and strong electric field enhancements due to the coastal conductivity contrast.

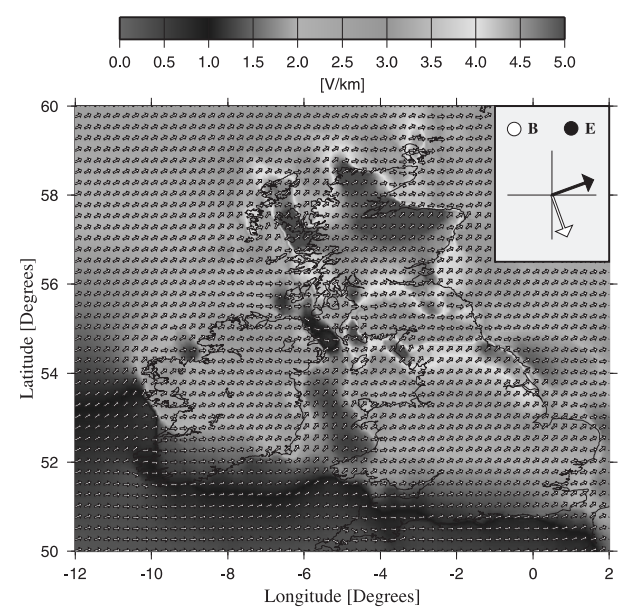

Fig. 2. Surface electric field throughout the UK estimated at 21:20UT assuming a driving period of $360 \mathrm{~s}$. The colour and arrows represent the E-field amplitude $[\mathrm{V} / \mathrm{km}]$ and azimuth respectively. The inset shows the azimuth of the primary geomagnetic field, and secondary electric field for a simple 1D model. After Thomson et al. (2005).

The Pre-Cambrian Shield is a highly resistive feature of Canadian geology. MT soundings have been made at many sites throughout Canada as part of the Lithoprobe project (e.g. Ferguson et al., 2005). Boteler (2001) used published MT soundings to determine regional conductivity models applicable to determining GIC in the five largest power systems in Canada. Fernberg et al. (2007) attributed anomalously large pipe to soil potentials to the lateral boundary between the shield and the relatively conductive Paleozoic rocks on the shield's eastern margin.

\section{EM Field Modelling}

The calculation of the geoelectric field at the Earth's surface normally comprises two main steps. First, specifying or determining the primary geomagnetic field responsible for induction. Second, calculation of the induced EM fields. In this Section we first outline how the geomagnetic disturbance can be modelled in regional studies. Then we highlight how the geoelectric field (since this is of primary importance in GIC studies) may be calculated.

\subsection{Modelling the geomagnetic disturbance field}

The characteristic feature of high geomagnetic latitudes is large and rapid temporal change, and strong spatial inhomogeneity, in the rate of change of the primary geomagnetic field (e.g. Pulkkinen and Viljanen, 2007). For example, Figure 3 illustrates the strong spatial inhomogeneity of the horizontal magnetic field rate of change just prior (20:06:30UT) to the power 
grid blackout experienced in Sweden at 20:07UT (Pulkkinen et al., 2005b) while the ground magnetic field is relatively smooth at 20:07:00UT.
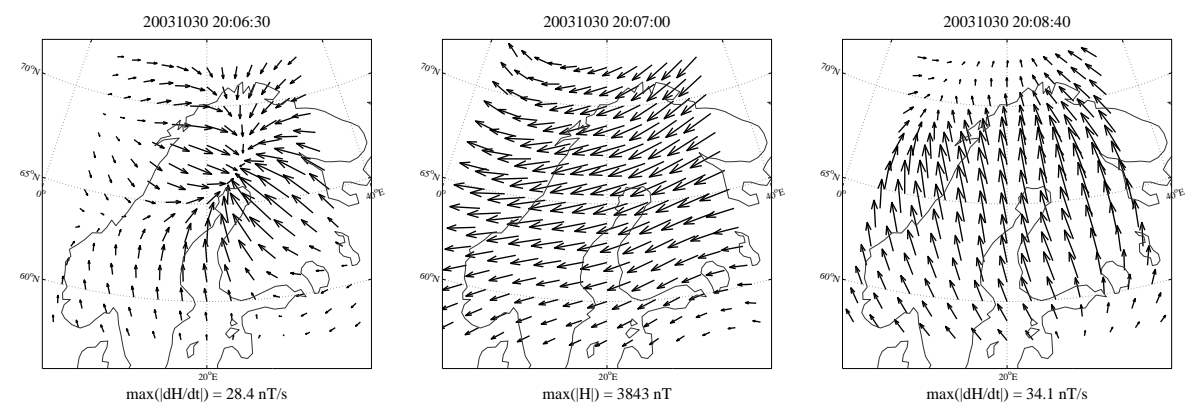

Fig. 3. The time derivative of the horizontal ground magnetic field at 20:06:30UT (left), equivalent ground currents formed by rotating the ground magnetic field at 20:07:00UT (middle) and the time derivative of the horizontal ground magnetic field at 20:08:40UT (right). The sampling rate of the geomagnetic field was 10s, and the measurements were interpolated onto a uniform grid using the SECS method.

The geomagnetic disturbance field is primarily of ionospheric origin. Geomagnetic disturbances are often represented using ionospheric equivalent currents. However, it is possible to try to predict the geomagnetic field at the Earth's surface directly from solar wind data (e.g. Gleisner and Lundstedt, 2001a,b; Weigel et al., 2003).

Models of idealized geomagnetic disturbances (e.g. a westward traveling surge) have been used to investigate the occurrence of GIC (e.g. Viljanen et al., 1999). The representation of the geomagnetic field using Spherical Elementary Currents Systems (SECS), introduced and validated by Amm (1997) and Pulkkinen et al. (2003a) respectively, means that recent studies have considered particular geomagnetic storm events and used ground based magnetometer data to derive equivalent ionospheric currents (e.g. Pulkkinen et al., 2003b) or interpolated maps of the ground magnetic field; see Figure 3 for example. SECS can also be used with a single magnetometer chain (Vanhamaki et al., 2003).

\subsection{Determining the geoelectric field}

The 'plane-wave' model (which forms the basis of the MT method) is commonly employed to calculate the geoelectric field. The simplest realization of the plane-wave method is a primary field that propagates vertically downward into an Earth of uniform or layered conductivity. The appeal is its simplicity, and its remarkable success (e.g. Viljanen et al., 2004).

The Complex Image Method (CIM) is an approximate method for calculating the EM fields at the Earth's surface (e.g. Boteler and Pirjola, 1998). Pulkkinen et al. (2003a) combined SECS and CIM; Viljanen et al. (2004) demonstrated that using CIM it is now possible to calculate quickly the geoelectric field using realistic representations of ionospheric current sources over a given region. They also demonstrated that the CIM method is accurate enough for the purpose of GIC studies despite using the plane-wave surface impedance. 
McKay and Whaler (2006) have shown that it is possible to use MT and GDS response functions to estimate directly the electric and magnetic fields throughout a region, rather than relying solely on conductivity models. Their study was limited to a single central period, however, where array (or the spatial coverage is good) MT and/or GDS data are available then the method is applicable to GIC.

3D Earth conductivity models have been applied in GIC research, but they have yet to be implemented in a practical sense; see for example Beamish et al. (2002) and Thomson et al. (2005). Pulkkinen and Engels (2005) used the 3D volume code of Avdeev et al. (2002), and the method of SECS to include both a non-uniform time-varying source field and 3D conductivity variations to study the effect of induction in the Earth on estimates of ionospheric equivalent currents. Significant induction effects were observed e.g. overestimation of up to $30 \%$ of the main ionospheric current flow amplitude, which increases away from the main current flow.

Pulkkinen et al. (2007) show that it is possible to estimate the MT surface impedance using GIC and geomagnetic observatory data. Therefore, Earth conductivity models which optimally describe the link between magnetic variations and GIC are being developed.

\section{Summary}

Significant progress in both Earth conductivity and EM modelling has been made in the last ten years, much of it relevant to the problem of the space weather impact on technological systems such as power networks and pipelines. In this short review we have summarised new findings on the geophysics relevant to the ground effects of space weather, and provided a reference list for more detailed reading. Future challenges with particular regard to GIC are the fast calculation of the EM fields using 3D Earth models, and developing an understanding of the level of model detail required. In conclusion we note that future planned satellite magnetometry missions, in particular the ESA mission SWARM, will likely provide even greater insights into the geophysical properties of the Earth and its environs, with clear benefit to the scientific and engineering communities interested in the ground effects of space weather.

\section{References}

Amm, O.: Ionospheric elementary current systems in spherical coordinates and their application. 49, 947-955 (1997).

Avdeev, D.B., Kuvshinov, A.V., Pankratov, O.V. and Newman, O.: Three dimensional induction logging problems, Part 1: an integral equation solution and model comparisons. Geophysics, 67(2),412-426 (2002).

Beamish D., Clark, T.D.G., Clarke E., and Thomson A.W.P.: Geomagnetically induced currents in the UK: geomagnetic variations and surface electric fields. J. Atmos. Sol. Terr. Phys., 64,1779-1792 (2002).

Boteler, D.H.: Assessment of geomagnetic hazard to power systems in Canada. Natural Hazards, 23, 101-120 (2001).

Boteler, D. and Pirjola, R.J.: The complex-image method for calculating the magnetic and electric fields produced at the surface of the Earth by the auroral electrojet. Geophys. J. Int., 132,31-40 (1998). 
Engels, M., Korja, T., and the Bear Working Group: Multisheet modelling of the electrical conductivity structure in the Fennoscandian Shield. Earth Planets Space, 54, 559-573 (2002).

Everett M.E., Constable, S. and Constable, C.G.: Effects of near surface conductance on global satellite induction responses, Geophys. J. Int., 153, 277-286 (2003).

Ferguson I.J., Craven, J.A., Kurtz, R.D., Boerner, D.C., Bailey, R.C., Wu, X., Orellana, M.R., Spratt, J., Wennberg, G. and Norton A.: Geoelectric response of Archean lithosphere in the western Superior Province, central Canada, Phys. Earth Planet. Int., 150, 123-143 (2005).

Fernberg, P. A., Samson, C., Boteler, D. H., Trichtchenko, L. and Larocca, P.: Earth conductivity structures and their effects on geomagnetic induction in pipelines. Annales Geophysicae, 25, 207-218 (2007).

Gleisner, H., and Lundstedt, H.: A neural network-based local model for prediction of geomagnetic disturbances, J. Geophys. Res., 106, 8425-8434 (2001a).

Gleisner, H., and Lundstedt, H.: Auroral electrojet predictions with dynamic neural networks, J. Geophys. Res., 106, 24541-24550 (2001b).

Haak, V.: Anomalies of electrical conductivity in the Earth's crust and upper Mantle. In: Fuchs, K. and Soffel, H. (ed), Landolt-Bornstein, New Series, 5/2b pp 397-436, Springer-Verlag, Berlin Heidelberg (1985)

Hjelt, S. E.: Regional EM studies in the 80s. Surv. Geophys., 9, 349-387 (1988)

Korja, T.: How is the European lithosphere imaged by Magnetotellurics? submitted Surv. Geophys., (2007)

Korja, T., Engels, M., Zhamaletdinov, A.A., Kovtun, A.A., Palshin, N.A., Smirnov, M.Y., Tokarev, A.D., Asming, V.E., Vanyan, L.L., Vardaniants, I.L., and the Bear Working Group: Crustal conductivity in Fennoscandia-a compilation of a databse on crustal conductance in the Fennoscandian Shield. Earth Planets Space, 54, 535-558 (2002)

Kuvshinov, A.: Global 3-D EM induction in the solid Earth and the oceans, in Electromagnetic sounding of the Earths interior, ed. Spichak V., Elsevier, Holland, Chapter 1, 4-24 (2007)

Kuvshinov, A. and Olsen, N.: A global model of mantle conductivity derived from 5 years of CHAMP, Ørsted and SAC-C magnetic data, Geophys. Res. Lett., 33, L18301, (2006).

Kuvshinov, A., Sabaka, T. and Olsen, N.: 3-D electromagnetic induction studies using the Swarm constellation: Mapping conductivity anoamlies in the Earth's mantle, Earth Planets Space, 58, 417-427 (2006).

Laske, G. and Masters, G., A global digital map of sediment thickness. EOS Trans. AGU, 78(46) (1997).

Lehtinen, M. and Pirjola, R., Currents produced in earthed conductor networks by geomagnetically induced currents. Annales Geophysicae, 3,4,479-484 (1985).

McKay, A.J. and Whaler, K.A.: The electric field in northern England and southern Scotland: implications for geomagnetically induced currents. Geophys. J. Int., 167, 613-625 (2006).

Olsen, N. and Kuvshinov A.: Modelling the ocean effect of geomagnetic storms: Earth, Planets and Space, 56, 525-530 (2004).

Pulkkinen, A., Pirjola, R., Boteler, D., Viljanen, A. and Yegorov, I: Modelling of space weather effects on pipelines. J. Appl. Geophys., 48, 233-256 (2001).

Pulkkinen, A., Amm, O., Viljanen, A. and the Bear Working group: Ionospheric equivalent current distributions determined with the method of spherical elementary current systems. J. Geophys. Res., 108(A2), 1053 (2003a).

Pulkkinen, A., Amm, O., Viljanen, A. and the Bear Working group: Separation of the geomagnetic variation field into parts of external and internal parts using the spherical elecmenatry currents system method. Earth Planets Space, 55, 117-129 (2003b).

Pulkkinen, A. and Engels, M.: The role of 3D geomagnetic induction in the determination of the ionospheric currents from ground-based data. Annales Geophysicae, 23, 909-917 (2005). 
Pulkkinen, A., Lindahl, S., Viljanen, A. and Pirjola, P.: Geomagnetic storm of 2931 October 2003: Geomagnetically induced currents and their relation to problems in the Swedish high-voltage power transmission system. Space Weather, 3, doi: 10.1029/2004SW000123 (2005b).

Pulkkinen, A., Viljanen, A. and Pirjola, P.: Determination of ground conductivity and system parameters for optimal modeling of geomagnetically induced current flow in technological systems. Earth Planets Space, submitted (2007).

Pulkkinen, A. and Viljanen, A.: The complex spatiotemporal dynamics of ionospheric currents. In: XXX(ed) COST724 Final Report. XXX (2007).

Purucker, M.: Magnetic anomaly map of the world. EOS Trans. AGU, 88(25), (2007).

Schwarz, G.: Electrical conductivity of the Earth's crust and upper mantle. Surv. Geophys., 11, 133-161 (1990)

Semenov, V. Yu and Jozwiak W.: Model of the geoelectrical structure of the mid and lower mantle in the Europe-Asia region. Geophys. J. Int., 138, 549-552 (1999).

Thomson, A.W.P., McKay, A.J., Clarke, E., Reay, S.J.: Surface electric fields and geomagnetically induced currents in the Scottish Power grid during the 30 October 2003 geomagnetic storm.Space Weather - The International Journal of Research and Applications, 3, Art. No. S11002 (2005).

Vanhamaki H, Amm O, Viljanen A: One-dimensional upward continuation of the ground magnetic field disturbance using spherical elementary current systems. Earth Planets Space, 55, 613-625 (2003).

Viljanen, A. and Amm, O. and Pirjola, R.: Modelling geomagnetically induced currents during different ionospheric situations. J. Geophys. Res., 104, 29,059-28,071 (1999).

Viljanen, A., A. Pulkkinen, O. Amm, R. Pirjola, T. Korja and BEAR Working Group: Fast computation of the geoelectric field using the method of elementary current systems and planar Earth models, Ann. Geophys., 22, 101-113, (2004)

Vozár, J., Semenov, V.Y., Kuvshinov, A.V. and Manoj, C.: Updating the map of Earth's surface conductance. EOS Trans. AGU, 33(15), (2006).

Weigel, R. S., Klimas, A.J. and Vassiliadis,D.: Solar wind coupling to and predictability of ground magnetic fields and their time derivatives, J. Geophys. Res., 108, 1298 (2003). 